\title{
Inverse design of nanostructured surfaces for color effects
}

\author{
Andkjær, Jacob Anders; Johansen, Villads Egede; Friis, Kasper Storgaard; Sigmund, Ole
}

Published in:

Optical Society of America. Journal B: Optical Physics

Link to article, DOI:

10.1364/JOSAB.31.000164

Publication date:

2014

Document Version

Publisher's PDF, also known as Version of record

Link back to DTU Orbit

Citation (APA):

Andkjær, J. A., Johansen, V. E., Friis, K. S., \& Sigmund, O. (2014). Inverse design of nanostructured surfaces for color effects. Optical Society of America. Journal B: Optical Physics, 31(1), 164-174.

https://doi.org/10.1364/JOSAB.31.000164

\section{General rights}

Copyright and moral rights for the publications made accessible in the public portal are retained by the authors and/or other copyright owners and it is a condition of accessing publications that users recognise and abide by the legal requirements associated with these rights.

- Users may download and print one copy of any publication from the public portal for the purpose of private study or research.

- You may not further distribute the material or use it for any profit-making activity or commercial gain

- You may freely distribute the URL identifying the publication in the public portal

If you believe that this document breaches copyright please contact us providing details, and we will remove access to the work immediately and investigate your claim 


\title{
Inverse design of nanostructured surfaces for color effects
}

\author{
Jacob Andkjær, Villads Egede Johansen, Kasper Storgaard Friis, and Ole Sigmund* \\ Technical University of Denmark, Department of Mechanical Engineering, 2800 Kgs. Lyngby, Denmark \\ ${ }^{*}$ Corresponding author: sigmund@mek.dtu.dk
}

Received May 28, 2013; revised November 27, 2013; accepted November 27, 2013; posted December 2, 2013 (Doc. ID 191305); published December 24, 2013

\begin{abstract}
We propose an inverse design methodology for systematic design of nanostructured surfaces for color effects. The methodology is based on a 2D topology optimization formulation based on frequency-domain finite element simulations for $E$ and/or $H$ polarized waves. The goal of the optimization is to maximize color intensity in prescribed direction(s) for a prescribed color (RGB) vector. Results indicate that nanostructured surfaces with any desirable color vector can be generated; that complex structures can generate more intense colors than simple layerings; that angle independent colorings can be obtained at the cost of reduced intensity; and that performance and optimized surface topologies are relatively independent on light polarization. ㄷ 2013 Optical Society of America OCIS codes: (230.1950) Diffraction gratings; (230.4170) Multilayers; (310.6628) Subwavelength structures, nanostructures; (330.1690) Color; (330.7326) Visual optics, modeling.

http://dx.doi.org/10.1364/JOSAB.31.000164
\end{abstract}

\section{INTRODUCTION}

Structural colors are caused by interference effects from light interaction with nanostructures rather than by pigments. The vivid colors generated by the nanostructures are evident in many animals and plants [1], e.g., the Morpho butterfly displays a blue color due to a tree-like multilayer structure [2] and the Papilio palinurus displays a bright green color [3]. Even though structural color effects have been of scientific interest for centuries [4], renewed interest has been sparked in recent years due to advances in nanotechnology.

Particularly the wings of the Morpho butterfly, as already mentioned, have been studied intensively for their optical properties and structural coloration. With the development of the electron microscope, it has been possible to observe the periodic ridge structure giving rise to the structural color. The ridge structure has a treelike shape that resembles a multilayer structure. The multilayer structure is tuned such that a reflection band occurs for the "blue" wavelengths. However, the color appears blue in a too-wide angular range compared to traditional grating and multilayer theories. The explanation is the height randomization of individual ridges, which destroys interference and hence causes smooth angular color appearance. In [5] a numerical analysis is conducted on the $3 \mathrm{D}$ nanostructure, which is the source of the blue color of the Morpho butterfly wings. Further studies on numerical modeling of Morpho structures and their randomness can be found in [6] , and a methodology for rendering of Morpho wings can be found in [7].

Much research has been centered on analyzing and artificially replicating the structures found in nature-so-called biomimetics or biomimicry [3,8]. However, in the present work we take another approach. Instead of mimicking what nature produced, we define the inverse design problem: find a dielectric nanostructure that displays a prescribed color effect. In this way we are not limited to what can be found in nature-we should be able to produce any desired color effect. The work is motivated by the developments in nanotechnology, improving the possibilities for achieving submicrometer features on surfaces also in large batch sizes. Examples of future applications are within design of nondegradable colors [8] and paint-free productions [9].

In previous studies [10-12], a method based on topology optimization [13] has been formulated for designing nanostructured surfaces with extreme reflection or transmission properties. Here we extend [12] by including a near- to farfield transformation of the reflected light and converting the scattered far-field spectrum to colors. With this extension, we obtain a methodology based on topology optimization for designing nanostructured multilayered surfaces displaying prescribed structural color properties.

Topology optimization is an inverse design methodology based on repeated analyses (finite element or finite difference analysis in frequency or time domain), gradient computations and material redistribution based on mathematical programming concepts. Topology optimization was originally developed for mechanical design problems $[\underline{13}, \underline{14}]$ but has since then been extended to a number of other physics areas, including nanophotonics [15-17], antennas [18], and metamaterials [19].

The power of the topology optimization method is that it can suggest novel geometries without any geometrical restrictions. Obviously, this extreme design freedom may also result in structures that are difficult to manufacture using currently available technologies. If the manufacturing technology and its limitations are known, one may introduce manufacturability as a part of the optimization problems as, e.g., seen in our previous work, where we introduced a connectivity constraint [11]. Obviously, imposing constraints on the geometrical freedom limits the achievable color response. To give the reader an idea of this aspect, we compare the performance of the 
designs achieved with full geometrical freedom with corresponding performances for designs that are limited to be simple layerings.

The rest of the paper is organized as follows. Section 2 describes the finite element modeling procedure, the nearto far-field mapping, the color-conversion scheme, as well as the randomization that suppresses diffraction effects. Section 3 describes the inverse design methodology, the optimization problem, and the numerical procedure. Section 4 discusses a range of optimized surface structures for different design goals, and Section $\underline{5}$ concludes on the work.

\section{MODELING OF COLOR-DISPLAYING NANOSTRUCTURED SURFACES}

\section{A. Numerical Model}

The numerical setup is similar to our previous study [12] but extended with a near- to far-field transformation as well as a color-conversion process. The computational domain is given in $2 \mathrm{D}$ and consists of three regions: an air region $\Omega_{A}$; a design region $\Omega_{D}$; and a bulk region $\Omega_{B}$, see Fig. 1 . Assuming invariance of the electromagnetic properties in the out-of-plane direction, Maxwell's equations simplify to the scalar Helmholtz equation. The scalar Helmholtz equation governs the physics for steady-state electromagnetic wave problems with a sinusoidal $E_{z}$ or $H_{z}$ polarized plane wave of angular frequency $\omega$ using $e^{j \omega t}$ to convert from phasor to time notation. Here we state the governing equation for an $E_{z}$ polarized wave. Equivalent equations for $H_{z}$ polarization are easily obtained by interchanging $E_{z} \leftrightarrow H_{z}$ and $\epsilon_{r} \leftrightarrow \mu_{r}$. The scalar Helmholtz equation is given as $[\underline{20}$, p. 9]

$$
\nabla \cdot\left(\mu_{r}^{-1} \nabla E_{z}\right)+k_{0}^{2} \epsilon_{r} E_{z}=0,
$$

where $\epsilon_{r}$ is the relative permittivity, $\mu_{r}$ is the relative permeability, and $k_{0}=\omega / c$ is the free-space wave number. The domain is truncated using perfectly matched layers

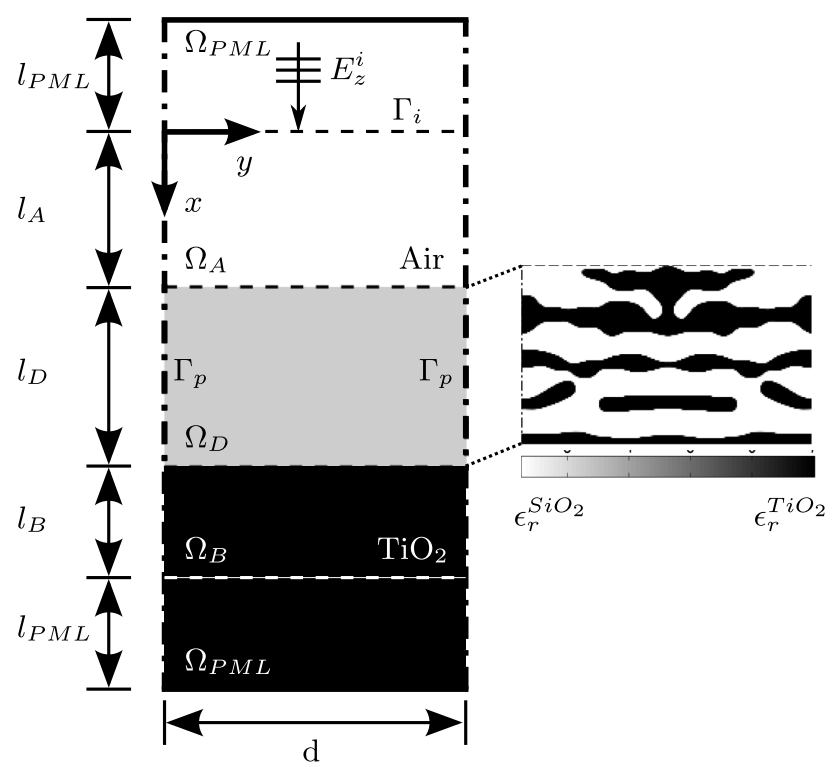

Fig. 1. Computational domain composed of an air region $\Omega_{A}$, a $\mathrm{TiO}_{2}$ region $\Omega_{B}$, and a design domain $\Omega_{D}$, where $\mathrm{SiO}_{2}$ and $\mathrm{TiO}_{2}$ is distributed to form the nanostructure. The periodicity of the structure is modeled with Bloch-Floquet boundary conditions at $\Gamma_{p}$.
(PML) [20] at the top and bottom boundaries. The governing equation in the $\Omega_{\mathrm{PML}}$ regions is

$$
\frac{\partial}{\partial x}\left(\frac{s_{y}}{s_{x}} \mu_{r}^{-1} \frac{\partial E_{z}}{\partial x}\right)+\frac{\partial}{\partial y}\left(\frac{s_{x}}{s_{y}} \mu_{r}^{-1} \frac{\partial E_{z}}{\partial y}\right)+k_{0}^{2} s_{x} s_{y} \epsilon_{r} E_{z}=0
$$

where $s_{x}$ and $s_{y}$ are complex functions of the position and govern the damping properties of the PML. The incident field $E_{z}^{i}$ is given as

$$
E_{z}^{i}=E_{z 0} \exp \left(-j k_{0} \sqrt{\mu_{r} \epsilon_{r}} \hat{\mathbf{k}} \cdot \mathbf{r}\right)
$$

where $E_{z 0}$ is the amplitude of the wave, $\hat{\mathbf{k}}=\left(\hat{k}_{x}, \hat{k}_{y}\right)^{T}$ is the normalized directional wave vector, and $\mathbf{r}=(x, y)^{T}$ is the spatial position vector. The incident wave is generated by a surface electric current density $\mathbf{J}_{s}$ on $\Gamma_{i}$ only having a $z$ component given by (see Appendix $\underline{\mathrm{A}}$ )

$$
J_{s z}=2 \cos \left(\theta_{\text {in }}\right) \sqrt{\frac{\epsilon_{0} \epsilon_{r} \mu_{r}}{\mu_{0}}} E_{z}^{i},
$$

where $\theta_{\text {in }}$ is the angle of the incoming wave to the normal of $\Gamma_{i}$. The periodic Bloch-Floquet boundary conditions on $\Gamma_{p}$ are given by

$$
E_{z}(x, d)=E_{z}(x, 0) \exp \left(-j k_{0} \sqrt{\mu_{r} \epsilon_{r}} d \sin \left(\theta_{\mathrm{in}}\right)\right) .
$$

The problem is solved using the finite element method (FEM) [20], and discretization details can be found in [12].

The field in close proximity and inside the nanostructure is found with the solution of the FEM problem. However, the reflected wave will most likely propagate a distance equal to many wavelengths before reaching the eye of an observer. Hence, the field of interest is the far field and not the near field found from the FEM solution. To accommodate this, a near- to far-field transformation is performed on the radial component of the scattered field based on Huygen's principle [20] and discarding any terms that decay faster than $1 /(\rho)^{1 / 2}$ where $\rho$ is the observation distance. The radial component of the scattered far-field $E_{z}^{s r}$ can be extracted from the near field using the following expression from [20]:

$$
\begin{aligned}
E_{z}^{s r}\left(\rho, \lambda, \theta_{\text {out }}\right) \approx & \sqrt{\frac{j k_{0}}{8 \pi \rho}} \exp \left(-j k_{0} \rho\right) \ldots \\
& \int_{0}^{d}\left[\left(\hat{n}_{y} \sin \left(\theta_{\text {out }}\right)+\hat{n}_{x} \cos \left(\theta_{\text {out }}\right)\right) E_{z}^{s} \ldots\right. \\
& \left.-\frac{1}{j k_{0}}\left(\hat{n}_{x} \frac{\partial E_{z}^{s}}{\partial x}+\hat{n}_{y} \frac{\partial E_{z}^{s}}{\partial y}\right)\right] \ldots \\
& \exp \left(j k_{0}\left(x \cos \left(\theta_{\text {out }}\right)+y \sin \left(\theta_{\text {out }}\right)\right)\right) \mathrm{d} y
\end{aligned}
$$

where $E_{z}^{s}=E_{z}-E_{z}^{i}$ is the scattered field and $\theta_{\text {out }}$ is the observation direction. Note that the line integral along the boundary $\Gamma_{i}$ in Eq. ( $\left.\underline{6}\right)$ is only defined along the $y$ axis because the line for $\Gamma_{i}$ is constant with respect to $x$. The normalized reflection factor $L\left(\lambda, \theta_{\text {out }}\right)$ in the direction $\theta_{\text {out }}$ of the scattered far-field is found as 


$$
L\left(\lambda, \theta_{\text {out }}\right)=\frac{\rho \lambda}{\cos \left(\theta_{\text {inc }}\right) \cos \left(\theta_{\text {out }}\right)} \frac{\left|E_{z}^{s r}\left(\rho, \lambda, \theta_{\text {out }}\right)\right|^{2}}{\left|E_{z}^{i}(\lambda)\right|^{2}},
$$

where $\theta_{\text {inc }}$ is the angle of the incoming wave to the normal of the surface. In the above equation, we have multiplied with $\rho$ in order to make the reflection factor independent of the observation distance.

The numerical model, including far-field transform, was tested against structures with analytical solutions such as a cylinder as well as the Morpho butterfly wing microstructure from [5].

\section{B. Color Conversion}

Since colors (of nonemitting objects) are only perceived when lit by a light source, the reflection of the dielectric nanostructure must be analyzed in a well-defined light environment. The so-called $D_{65}$ standard illuminant spectrum that resembles daylight [21] is chosen as the light source. The reflected spectrum is then converted to coordinates in the RGB color space by applying the color-matching functions [22] shown in Fig. $\underline{2}$ :

$$
\begin{aligned}
R\left(\theta_{\text {out }}\right) & =K_{c} \int_{380}^{760} D_{65}(\lambda) \bar{r}(\lambda) L\left(\lambda, \theta_{\text {out }}\right) \mathrm{d} \lambda \\
& \approx K_{c} \sum_{\lambda} D_{65}(\lambda) \bar{r}(\lambda) L\left(\lambda, \theta_{\text {out }}\right) \Delta \lambda, \\
G\left(\theta_{\text {out }}\right) & =K_{c} \int_{380}^{760} D_{65}(\lambda) \bar{g}(\lambda) L\left(\lambda, \theta_{\text {out }}\right) \mathrm{d} \lambda \\
& \approx K_{c} \sum_{\lambda} D_{65}(\lambda) \bar{g}(\lambda) L\left(\lambda, \theta_{\text {out }}\right) \Delta \lambda, \\
B\left(\theta_{\text {out }}\right) & =K_{c} \int_{380}^{760} D_{65}(\lambda) \bar{b}(\lambda) L\left(\lambda, \theta_{\text {out }}\right) \mathrm{d} \lambda \\
& \approx K_{c} \sum_{\lambda} D_{65}(\lambda) \bar{b}(\lambda) L\left(\lambda, \theta_{\text {out }}\right) \Delta \lambda,
\end{aligned}
$$

where $K_{c}$ is a scaling factor and the integration is approximated by summation for 20 discrete, equidistant wavelengths between 380 and $760 \mathrm{~nm}$. Note that the $D_{65}$ spectrum is taken into account in the expression, and hence FEM simulations

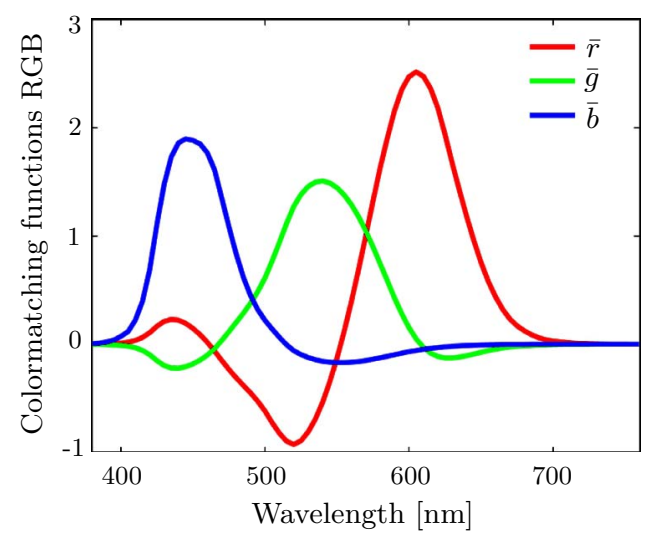

Fig. 2. Color-matching functions for finding the RGB channels of a light spectrum. The functions are derived using the $1931 \mathrm{CIE} 2^{\circ}$ standard observer weighting function [21] to obtain the XYZ tristimulus values and afterward converted to the RGB working space using a $D_{65}$ reference while following ISO 22028-1:2004. can be carried out with equal input intensity for each wavelength. The match among the color-matching function, reference illuminant, and light source further ensures that a reflected flat spectrum will appear white, as expected intuitively. The scaling factor $K_{c}$ is found such that the RGB values from the specular reflection of a fully reflecting surface are 1 . Note that this normalization does not guarantee that individual RGB numbers do not exceed unity or go below zero. This is due to the negative intervals of the color-matching functions (cf. Fig. 2).

\section{Suppressing Diffraction Effects}

Reflection from strictly periodic structures lit by a plane wave creates diffraction. Diffraction is constructive and destructive interference, resulting in light only being reflected at certain angles. These reflection angles are wavelength-dependent. This means that a perfectly periodic structure illuminated by white light as seen in Fig. 3(a) will reflect a rainbow pattern as illustrated in the reflected spectra of Fig. 3(b). This effect can, for example, be observed when looking at a compact disc (CD). The angles of reflection are determined by the period of the surface structure. To avoid this, random height displacements can be introduced to remove the interference effects and make it possible to predict the total reflected intensity as the reflected intensity from one "period." This has been shown in theory as well as in practice in $[6,23,24]$ and is a combination of random height variation and light incoherence, which obscure phase information in the reflected electromagnetic wave. Reference [5] demonstrates relevant random height distributions and [24] shows that a binary height distribution in practice is enough if the wanted color can be represented by a small band of wavelengths. The random height interval can in general be kept below half of the longest

(a)

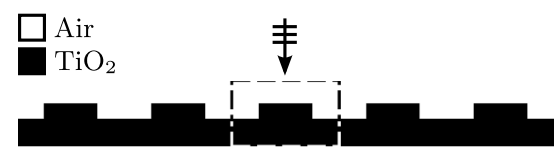

(b) $-80^{\circ}-60^{\circ}-40^{\circ}-20^{\circ} \quad 0^{\circ} \quad 20^{\circ} \quad 40^{\circ} \quad 60^{\circ} \quad 80^{\circ}$

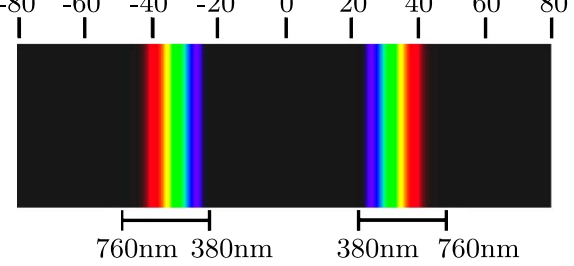

(c)

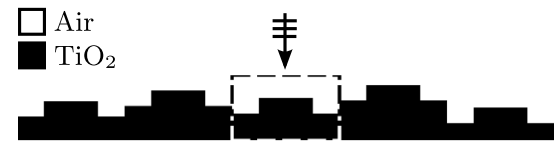

(d)

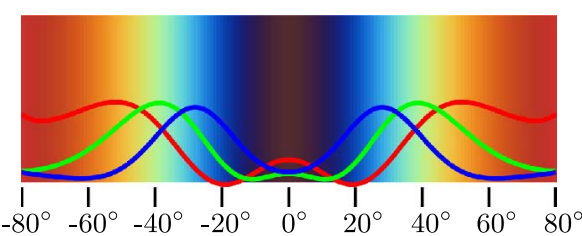

Fig. 3. Perfectly periodic nanostructure (a) with corresponding diffraction pattern (b) and a height randomized structure (c) with the diffraction effect eliminated (d). The display colors are exaggerated for the purpose of illustration. The area surrounded by the dashed line corresponds to one "unit cell." 
wavelength of interest corresponding to a phase change of $360^{\circ}$ at that wavelength.

The simulations are carried out assuming that a random variation can be applied afterward (see, e.g., [23]) as illustrated in Fig. 3(c), giving rise to the reflected spectra seen in Fig. 3(d), where all diffraction effects are removed. We will not deal with this aspect in further detail here, but assume that a random height variation as seen in [5] can be applied to make the response from one period of the structure appear similar to the response of an array of randomized cells.

\section{INVERSE DESIGN METHODOLOGY}

\section{A. Material Distribution by Topology Optimization}

Light can be reflected and/or refracted at interfaces between two media, depending on the spatial placement and distribution of the material properties $\left(\epsilon_{r}\right.$ and $\left.\mu_{r}\right)$. Hence, control of these material properties allows wave manipulation for various purposes, e.g., creating reflection bands for coloration. Here we work with a $\mathrm{TiO}_{2}$ substrate with a nanostructure composed of $\mathrm{TiO}_{2}$ and $\mathrm{SiO}_{2}$; however, the developed topology optimization methodology can handle any simple (linear, homogeneous, isotropic) material combinations including metallic structures [25] and also frequency-dependent material properties. The method works by varying the distribution of materials within a bounded design domain in order to optimize certain responses of the physical system. In the design domain $\Omega_{D}$ between the air and $\mathrm{TiO}_{2}$ substrate, the relative permittivity can be varied continuously on an element basis between the permittivity for $\mathrm{SiO}_{2}\left(\epsilon_{r}^{\mathrm{SiO}_{2}}=2.25\right)$ and $\mathrm{TiO}_{2}\left(\epsilon_{r}^{\mathrm{TiO}_{2}}=7.02\right)$. We employ a standard density-based topology optimization method [16] and restrict our investigations to nonmagnetic materials $\left(\mu_{r}=1\right)$. A continuous design variable $\gamma \in[0 ; 1]$ is introduced for each element in the design domain $\Omega_{D}$ and controls the element material properties. Here $\gamma_{e}=0$ corresponds to $\mathrm{SiO}_{2}$ and $\gamma_{e}=1$ corresponds to $\mathrm{TiO}_{2}$ :

$$
\epsilon_{r}\left(\gamma_{e}\right)=\epsilon_{r}^{\mathrm{SiO}_{2}}+\gamma_{e}\left(\epsilon_{r}^{\mathrm{TiO}_{2}}-\epsilon_{r}^{\mathrm{SiO}_{2}}\right) .
$$

The continuous design variable formulation allows us to solve the optimization problem with efficient gradient-based design updates [26]. In principle, the optimization may result in "gray scale" results, i.e., elements that neither correspond to $\mathrm{SiO}_{2}$ nor to $\mathrm{TiO}_{2}$; however, the robust design formulation developed in $[27,28]$ and also used in [12] ensures almost discrete designs through a continuation strategy. Symmetric solutions are ensured by mirroring of design variables.

\section{B. Optimization Problem}

The idea of the inverse design methodology is to find a nanostructure that displays a desired color effect in a specific angle or angular range. The desired color is given as a reference in the RGB color space $R G B_{r}=\left[R_{r}, G_{r}, B_{r}\right]$. The direction of the color vector $R G B\left(\theta_{\text {out }}\right)$ (for nonzero length) decides the color, and the length decides the color intensity. In order to create a desired color effect, it is crucial that the color vector from the nanostructure is parallel to the reference color vector. This is ensured by imposing a constraint on the normalized crossproduct between the actual and the prescribed color vectors. With the color vector constrained in the desired direction in the color space, the normalized intensity of the reflected light is maximized. Furthermore, a volume constraint is imposed to prevent congestion in the design domain. The optimization problem is formulated as a maxmin formulation for design problems involving a color effect in an angular range or different colors reflected in different directions simultaneously. The optimization maxmin problem maximizing the minimum intensity of several angular directions is formulated as

$$
\begin{array}{ll}
\max _{\gamma} & \Phi:=\min _{k=1, \ldots, N} \frac{\left|R G B\left(\theta_{\text {out }}^{k}\right)\right|^{2}}{\left|R G B_{r}^{k}\right|^{2}}, \\
\text { s.t. } & \frac{\left|R G B\left(\theta_{\text {out }}^{k}\right) \times R G B_{r}^{k}\right|^{2}}{\left|R G B_{r}^{k}\right|^{2}} \leq \tau^{2}, \quad k=1, \ldots, N, \\
& \frac{1}{V_{\Omega_{D}}} \int_{\Omega_{D}} \gamma \mathrm{d} \Omega_{D}-\beta \leq 0, \\
& 0 \leq \gamma \leq 1,
\end{array}
$$

where $k$ is the index of the $N$ angular directions, $\tau$ is the highest admissible value of the error between the actual and prescribed color vectors, $V_{\Omega_{D}}$ is the total volume of the design domain $\Omega_{D}$, and $\beta$ is the admissible volume fraction. The constraint on the color vector is introduced for each angular direction in the problem. The prescribed reference colors in the color constraints do not need to be the same but can be set to any desired RGB value. The average of the RGB values obtained from both $E_{z}$ and $H_{z}$ polarized waves is used in Eq. (12) for unpolarized color effects.

The design is updated iteratively using the gradient-based optimization routine method of moving asymptotes (MMA) [29]. The normalized intensity given as $\Phi$ in Eq. (12) is used as a performance measure for the nanostructures, and the sensitivities are obtained using the adjoint method [15]. The maxmin problem in Eq. (12) is in general a challenging problem due to non-differentiability of the min-function. To circumvent this, the problem is reformulated to the so-called bound-formulation, which is a standard conversion allowed by the MMA optimizer. The interested reader is referred to the MMA paper [29] and Svanberg's publicly available codes for further explanations.

The design procedure for black is constructed as a special case. The objective for black is to absorb or transmit the light for all visible wavelengths and thereby have zero reflection. Hence the maximum intensity $\Phi\left(\theta_{\text {out }}\right)$ at several angular directions is minimized in the optimization problem [cf. Eq. (12)] while using white as a reference color $\left(R_{r}=1, G_{r}=1\right.$, $\left.B_{r}=1\right)$. The lower the value of $\Phi$, the better the nanostructure is in displaying black.

The proposed procedure is implemented in MATLAB utilizing parallel computations for the frequency sweeps. Convergence is typically reached after 500-1000 material redistribution steps, where the main computational effort in each step consists in the forward and adjoint FEM solutions for the 20 frequencies. This effort may be significantly reduced using Padé approximants for the frequency sweeps [15], a feature we will implement in future versions of the code. Presently, one optimization runs overnight on an eight-processor computer.

\section{STRUCTURAL COLORS GENERATED BY TOPOLOGY OPTIMIZED NANOSTRUCTURES}

In this section, we demonstrate the procedure's ability to design nanostructured surfaces with tailored color properties for narrow and wider viewing angles, for polarized and 
(a)

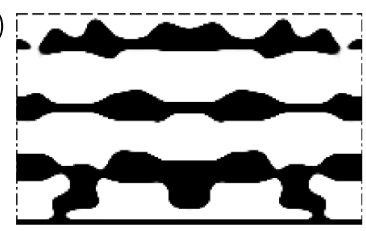

(b)

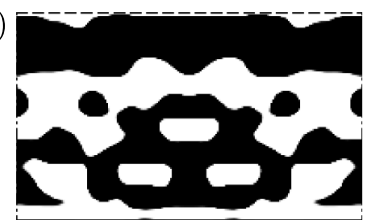

(c)

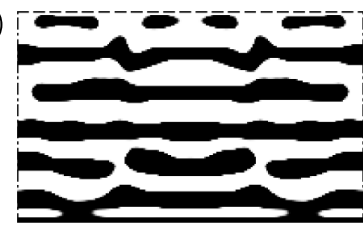

(d)

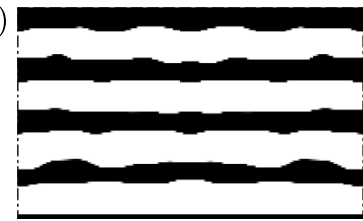

(e)

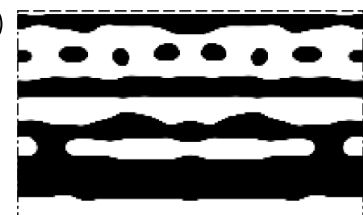

(f)

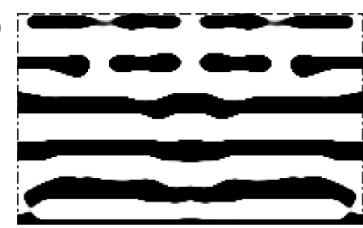

(g)

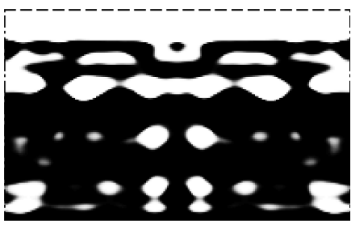

(h)

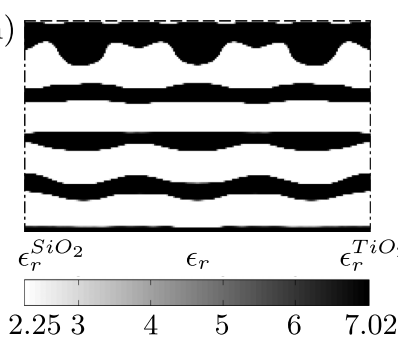

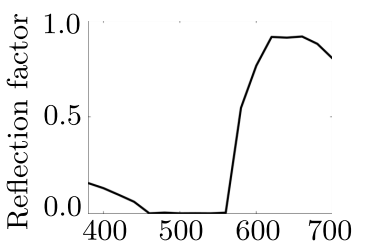

$\Phi=1.0305$
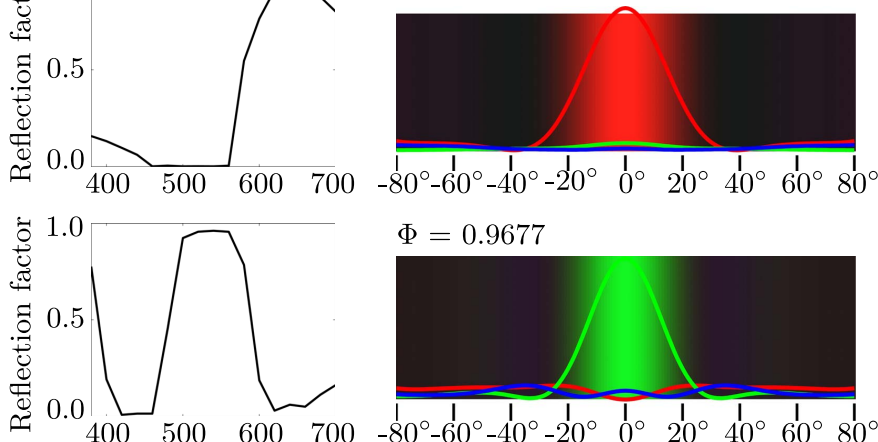

$\Phi=0.9677$
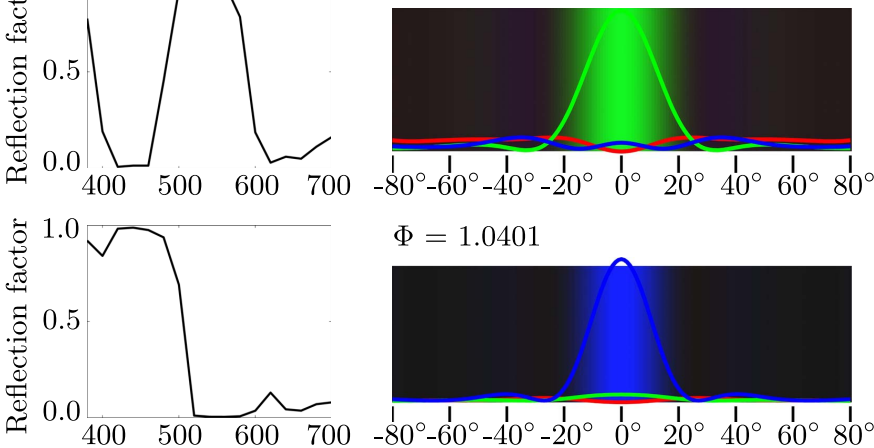

$\Phi=1.0401$
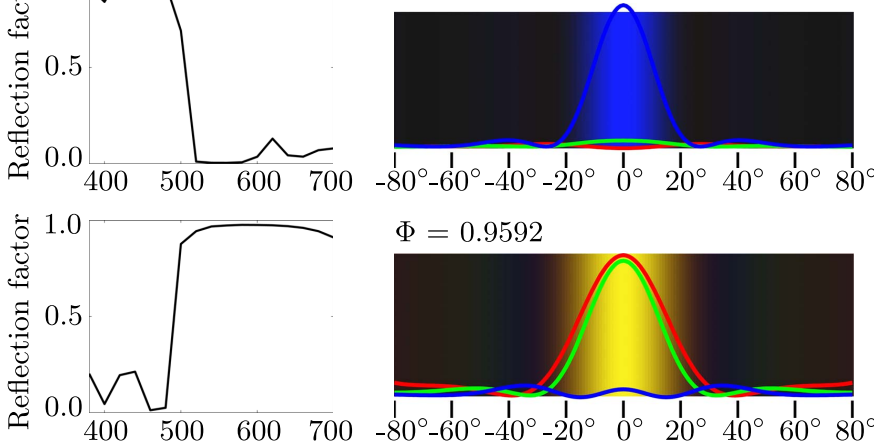

$\Phi=0.9592$
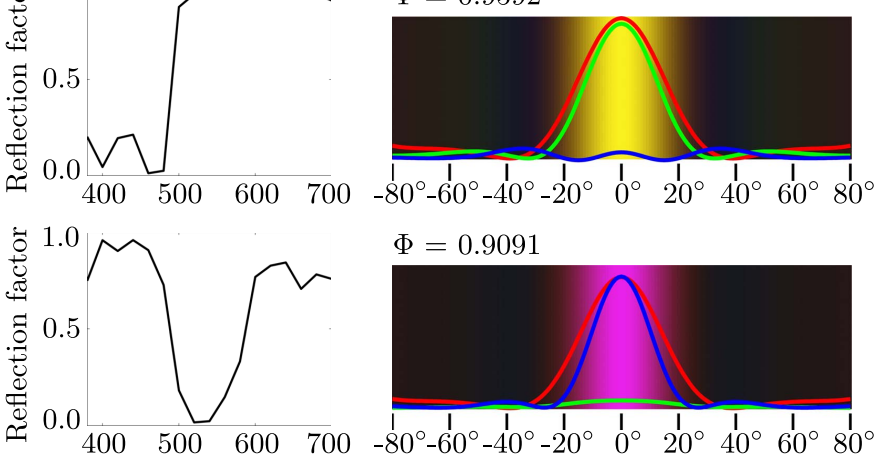

$\Phi=0.9091$
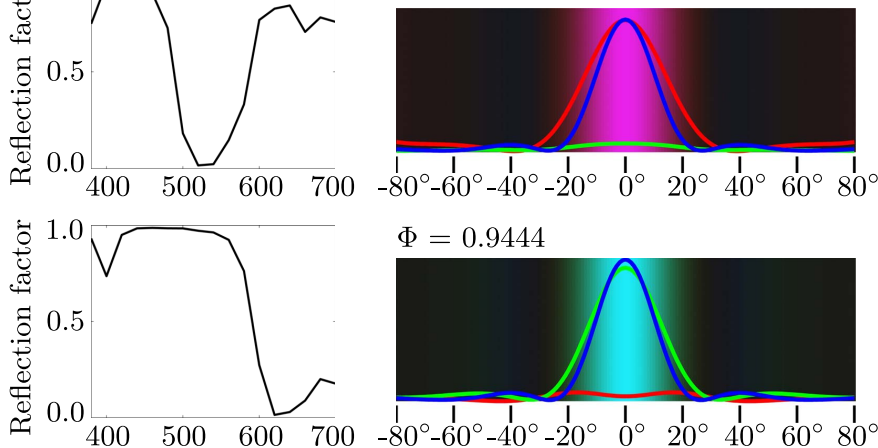

$\Phi=0.9444$
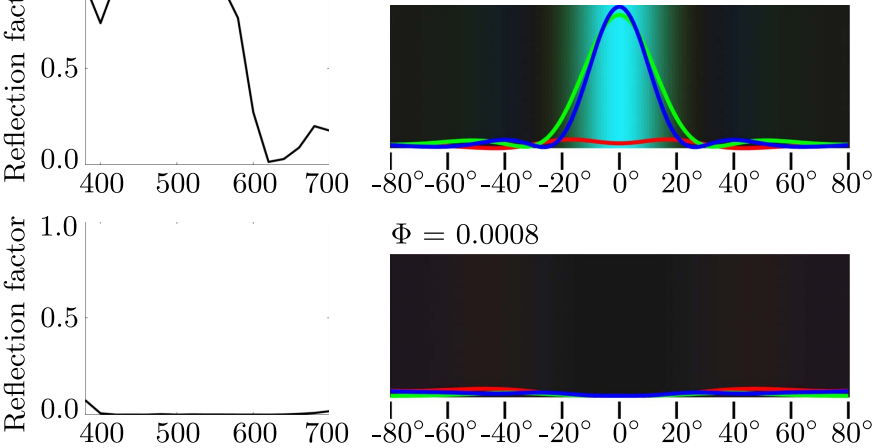

$\Phi=0.0008$
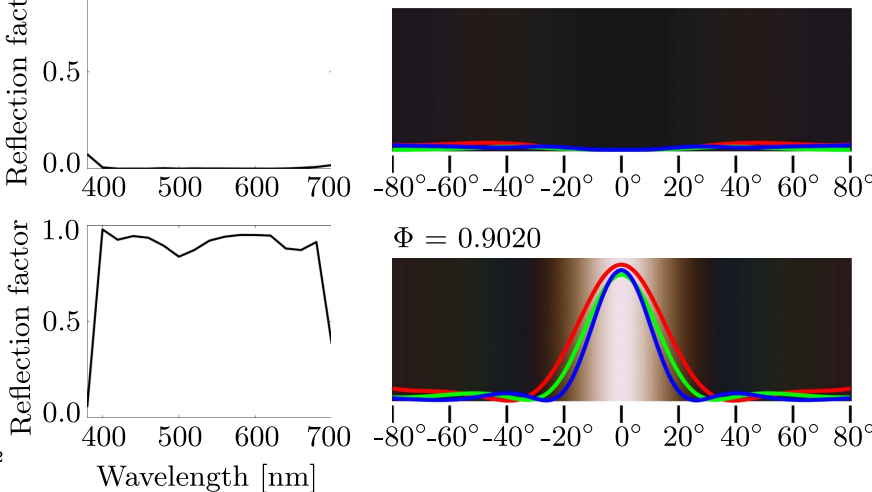

$\Phi=0.9020$

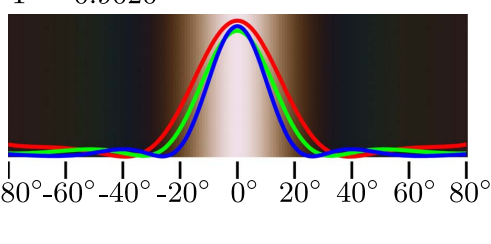

Fig. 4. Color optimization for $0^{\circ}$ observation angle. The left column shows the material distribution; the center column the frequency response seen from the observer; and the right column shows the angular color spectrum including individual color distributions. Structures (a)-(h) are optimized for red $\left(R G B_{r}=[1,0,0]\right)$, green $[0,1,0]$, blue $[0,0,1]$, yellow $[0,1,1]$, magenta $[1,0,1]$, cyan $[1,1,0]$, black $[0,0,0]$, and white $[1,1,1]$, respectively. 
non-polarized light, as well as for prescribed iridescent, i.e., angular dependent, color properties. The nanostructuring is obtained by a nanostructured mix of $\mathrm{SiO}_{2}$ and $\mathrm{TiO}_{2}$ on a $\mathrm{TiO}_{2}$ substrate.
We consider a periodically repeated symmetric design domain as indicated with gray in Fig. 1 . The period is $d=$ $1000 \mathrm{~nm}$ and the thickness is $l_{D}=600 \mathrm{~nm}$. The design domain is discretized with $120 \times 200$ finite elements and a maximum of (a)

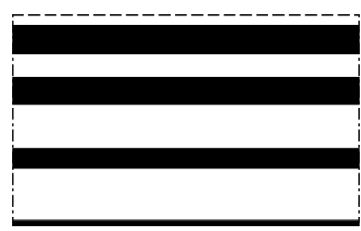

(b)

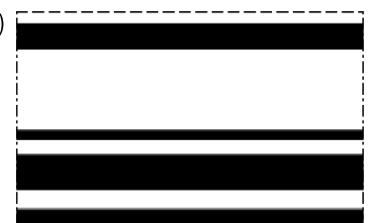

(c)

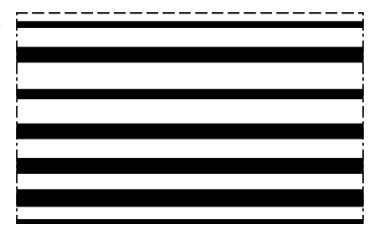

(d)

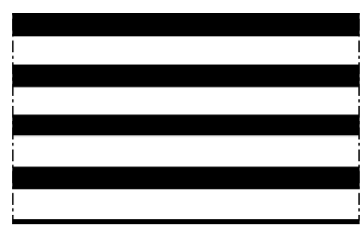

(e)

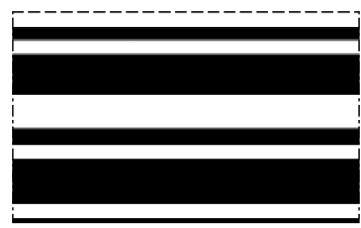

(f)

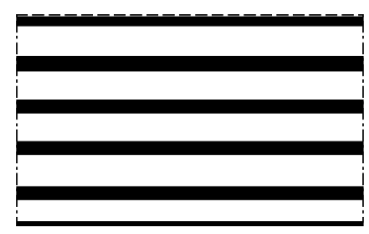

(g)

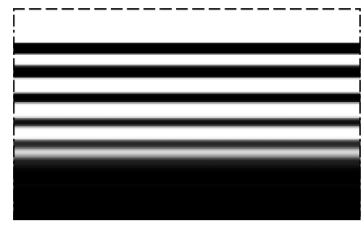

(h)

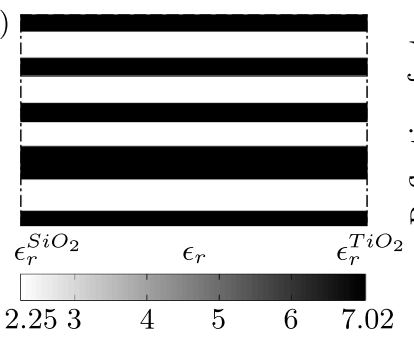

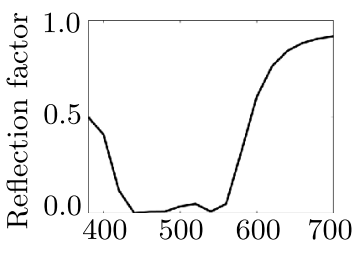

$\Phi=0.8239$
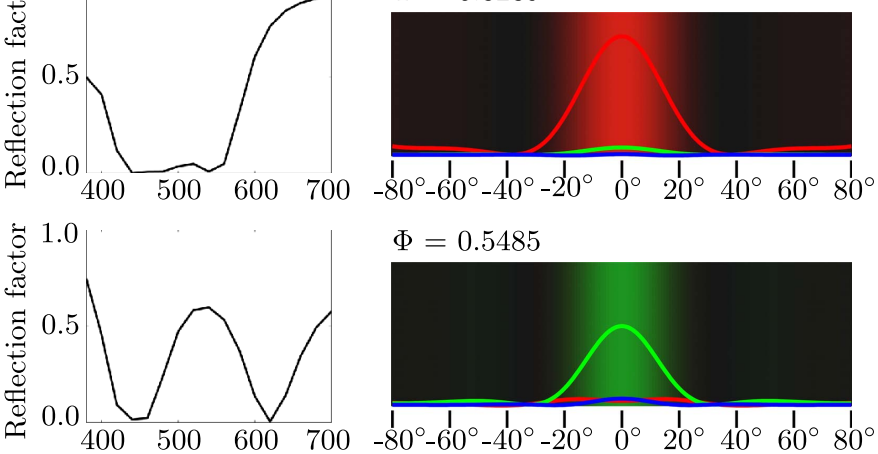

$\Phi=0.5485$
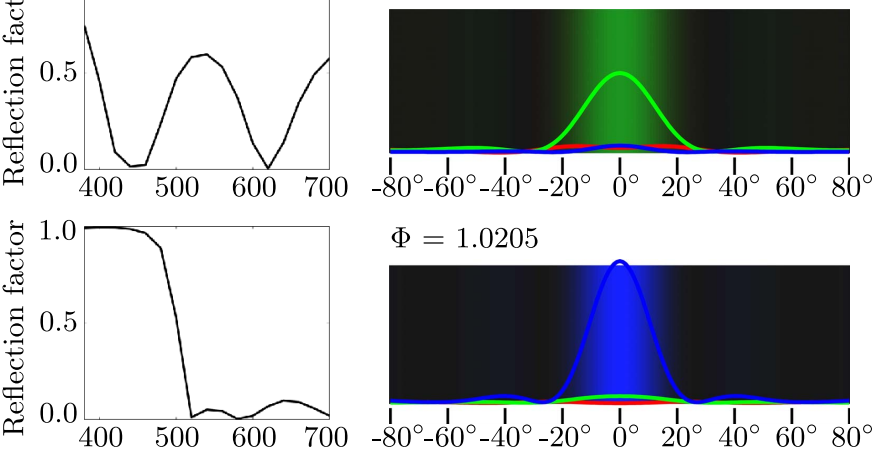

$\Phi=1.0205$
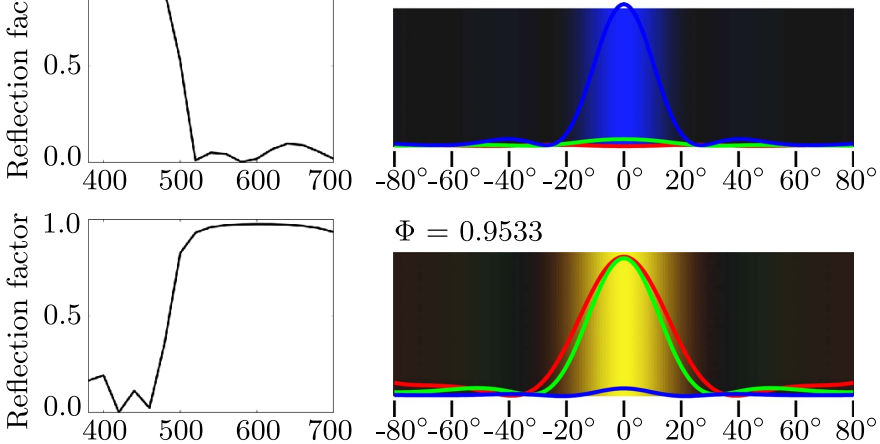

$\Phi=0.9533$
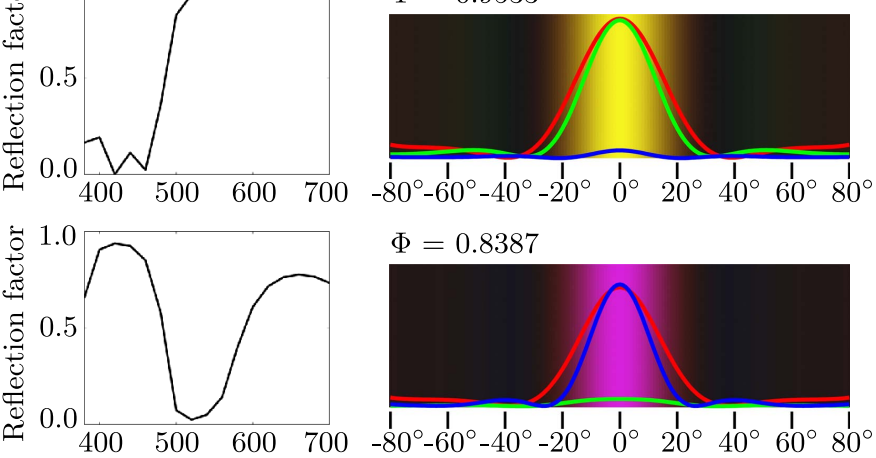

$\Phi=0.8387$
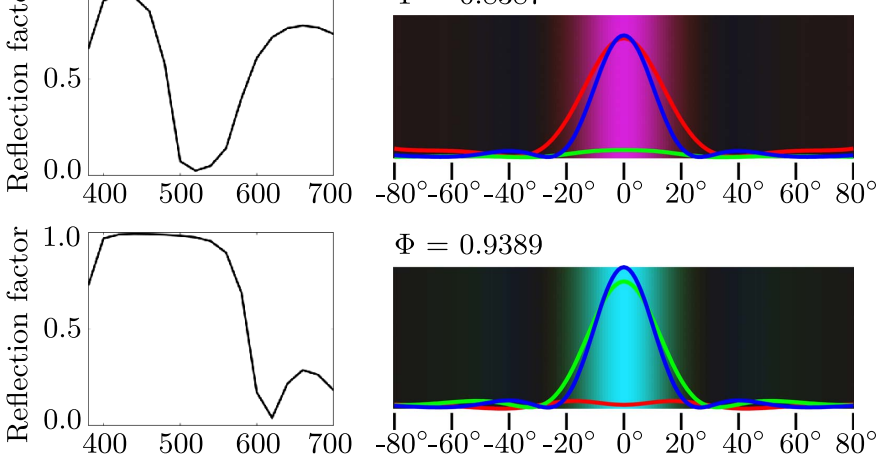

$\Phi=0.9389$
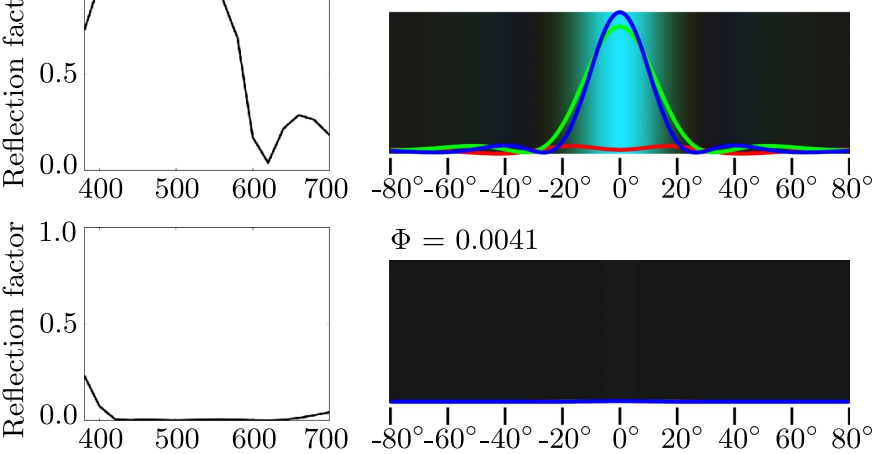

$\Phi=0.0041$
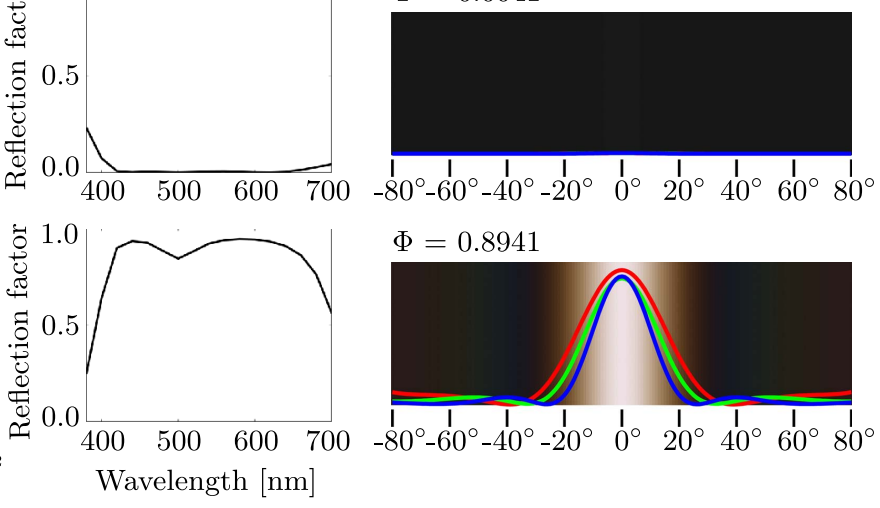

$\Phi=0.8941$

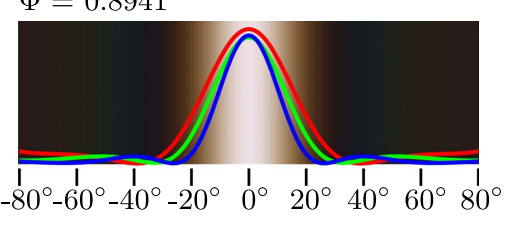

Fig. 5. Color optimization for $0^{\circ}$ observation angle but design freedom limited to simple layered Bragg-like structures. Other details can be read from the caption of Fig. 4 . 
$\beta=65 \%$ of $\mathrm{TiO}_{2}\left(\epsilon_{r}^{\mathrm{TiO}_{2}}=7.02\right)$ can be distributed in the $\mathrm{SiO}_{2}$ $\left(\epsilon_{r}^{\mathrm{SiO}_{2}}=2.25\right)$ base material. The choice of design domain thickness $l_{D}$ is chosen arbitrarily. A very small thickness will not be able to produce interesting color effects whereas a large thickness (i.e., much larger than the wavelength) will result in intense colors and be of less challenge to the optimizer.

We have used $\tau=0.05$ as default value in Eq. (12), i.e., we allow a $5 \%$ deviation from the desired color vector. Choosing a (a)

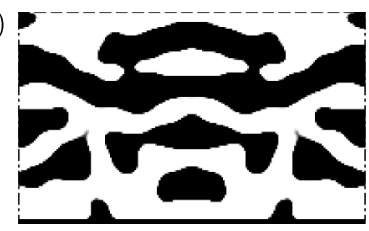

(b)

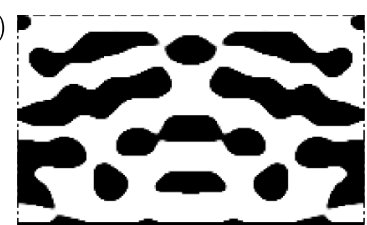

(c)

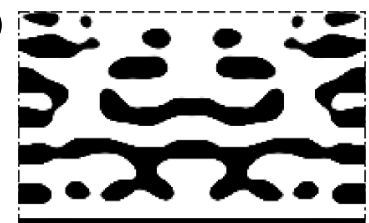

(d)

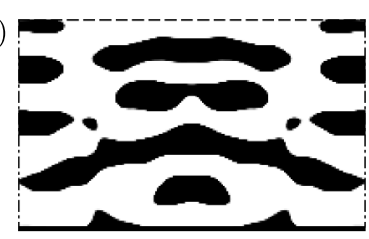

(e)

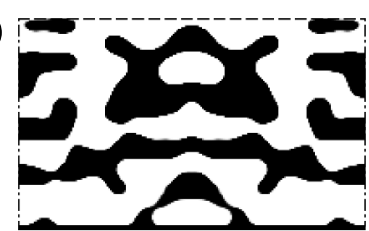

(f)

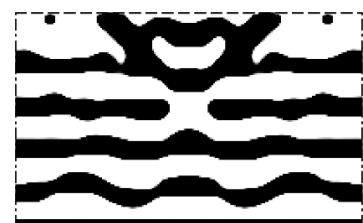

(g)

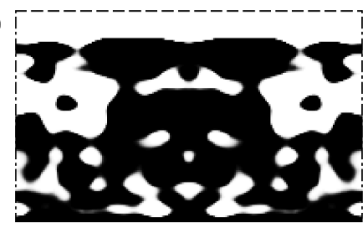

(h)

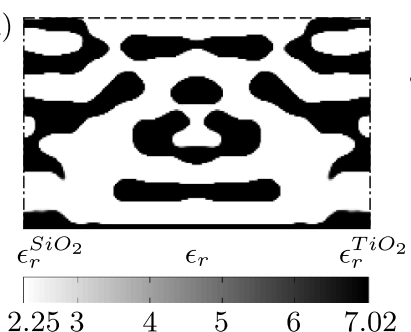

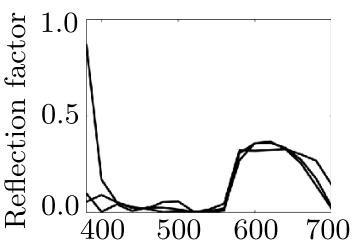

$\Phi=0.4282$
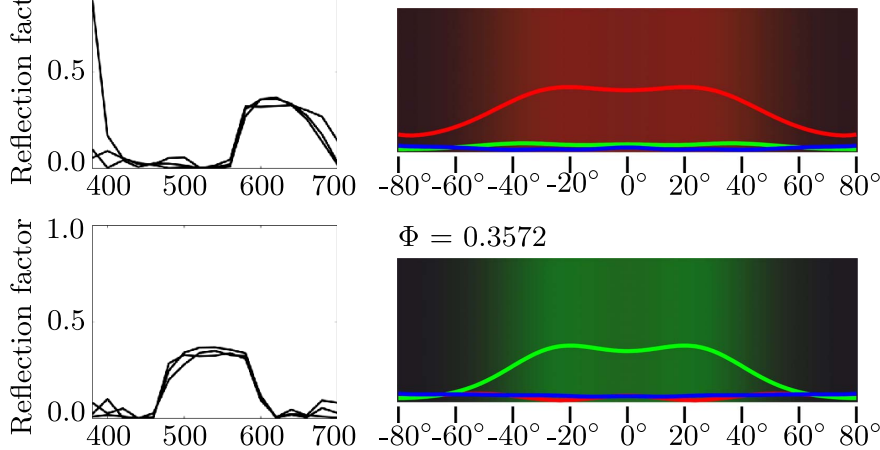

$\Phi=0.3572$
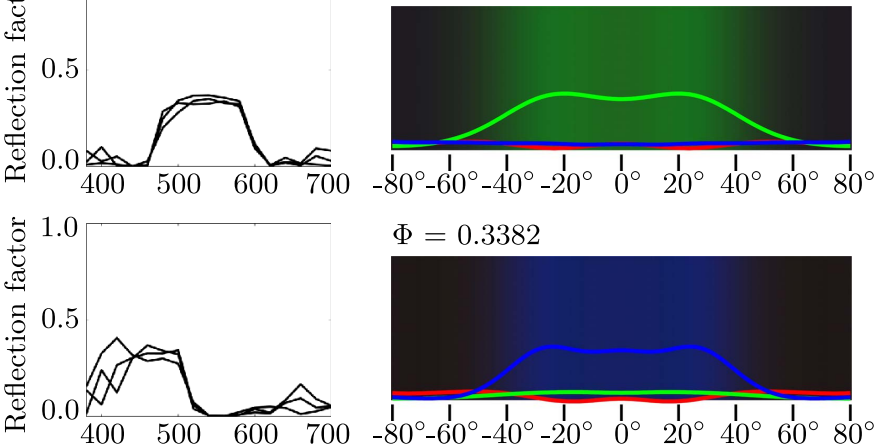

$\Phi=0.3382$
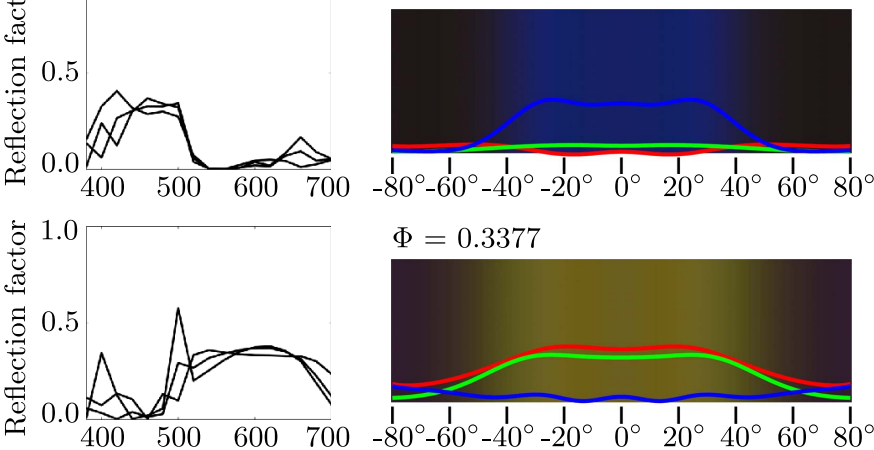

$\Phi=0.3377$
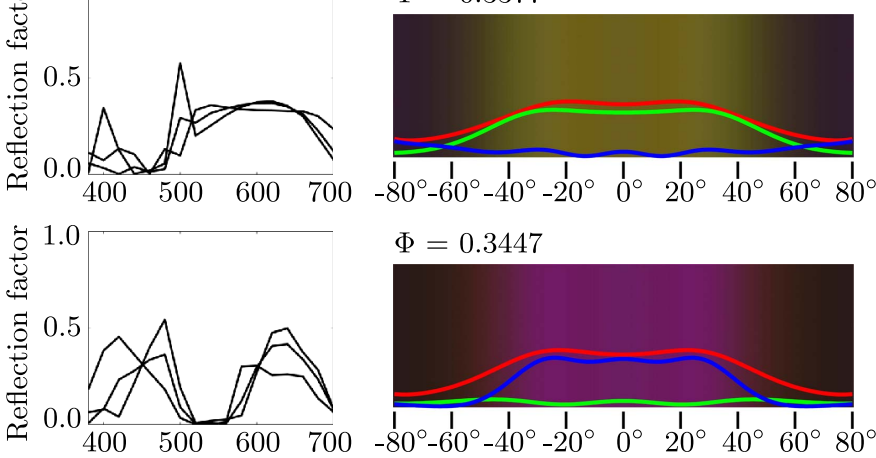

$\Phi=0.3447$
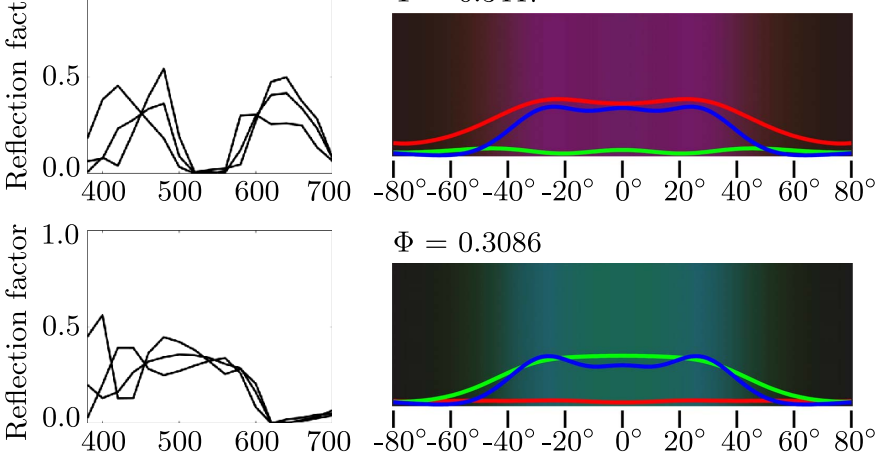

$\Phi=0.3086$
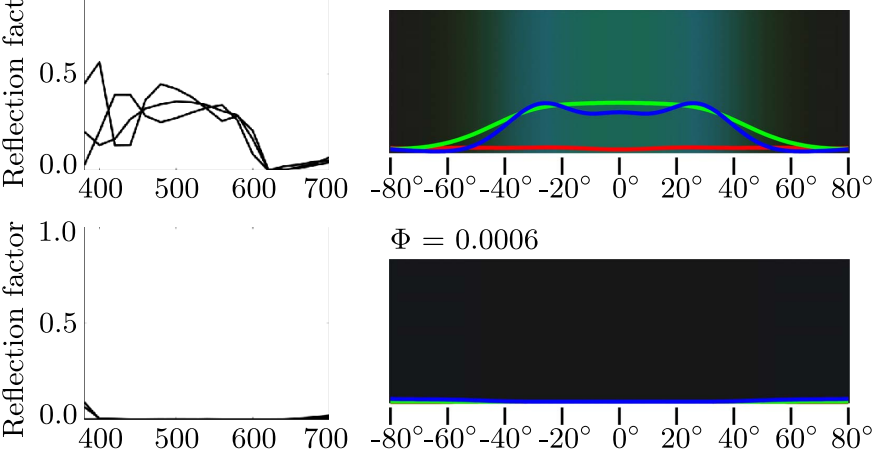

$\Phi=0.0006$
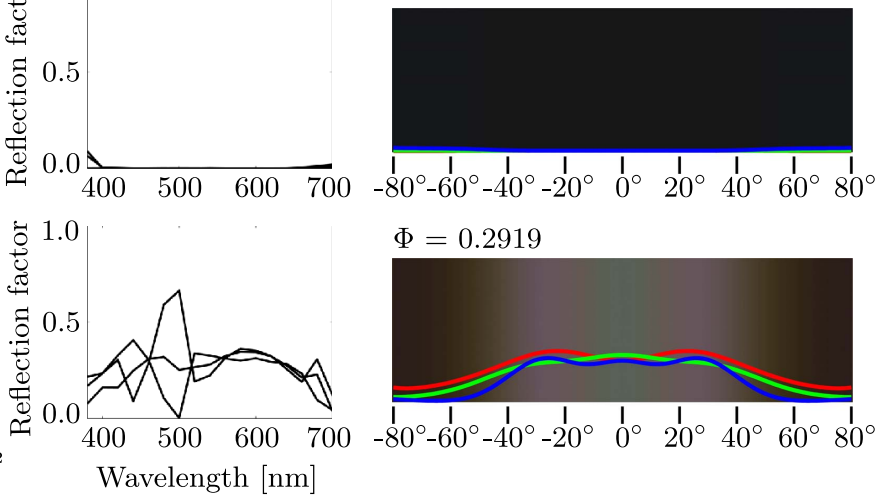

$\Phi=0.2919$

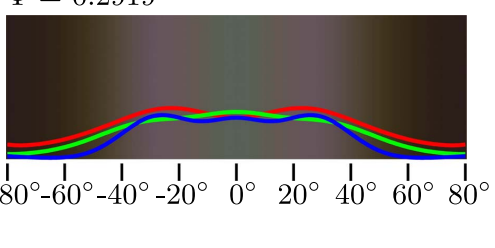

Fig. 6. Color optimization for $-30^{\circ}$ to $30^{\circ}$ observation angle with full design freedom. Other details can be read from the caption of Fig. 4 . The three curves in the reflection plots correspond to the reflection spectra for the three optimization angles $\left(0^{\circ}, 15^{\circ}\right.$, and $\left.30^{\circ}\right)$. 
Table 1. Objective Values for RGB Optimizations Corresponding to $0^{\circ}, 0^{\circ}$ Constrained to Simple Layerings and Wider Angular Spectrum $\left[-30^{\circ}, 30^{\circ}\right]^{a}$

\begin{tabular}{lcccccccccc}
\hline Objective & Figure & $\mathrm{R}$ & $\mathrm{G}$ & $\mathrm{B}$ & $\mathrm{Y}$ & $\mathrm{M}$ & $\mathrm{C}$ & $\mathrm{W}$ & $\mathrm{B}$ \\
\hline $0^{\circ}$ & $\underline{4}$ & 1.03 & 0.97 & 1.04 & 0.96 & 0.91 & 0.94 & 0.90 & 0.00 \\
$0^{\circ}$ layer & $\underline{5}$ & 0.82 & 0.55 & 1.02 & 0.95 & 0.83 & 0.93 & 0.89 & 0.00 & 0.31 \\
{$\left[-30^{\circ}, 30^{\circ}\right]$} & $\underline{6}$ & 0.43 & 0.36 & 0.34 & 0.34 & 0.34 & 0.34 & 0.29 & 0.00 \\
\hline
\end{tabular}

${ }^{a}$ The objective values correspond to Figs. $\underline{4}, \underline{5}$, and $\underline{6}$, respectively. Colors are abbreviated red (R), green (G), blue (B), yellow (Y), magenta (M), cyan (C), black (B), and white (W).

smaller value will make it harder for the optimizer to converge and will most probably not be visually perceptible anyway. As initial designs, we have used both pure $\mathrm{SiO}_{2}$ as well as a Bragg grating designed to have a reflection band at a wavelength corresponding to the desired color. When running the optimization for different initial designs we minimize the risk of finding a less desirable local optimum. In each case, we show the optimized design with the best performance out of the two initial designs. In most cases, the difference in performance is small anyway.

To begin with, we consider three different cases all with normal $\left(0^{\circ}\right)$ incident light: (1) optimizing the color in the (a)

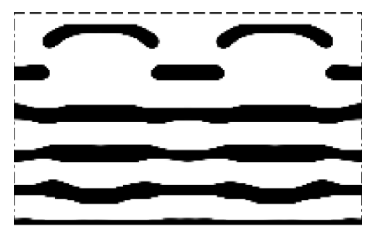

(b)

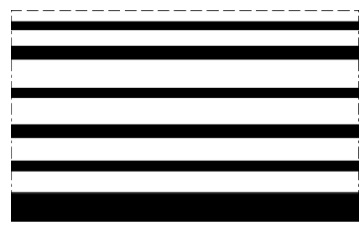

(c)

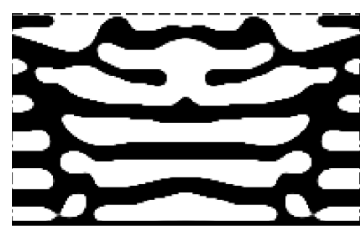

(d)

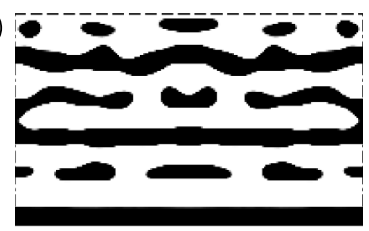

(e)

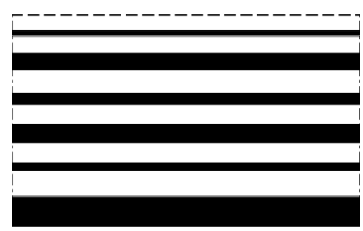

(f)

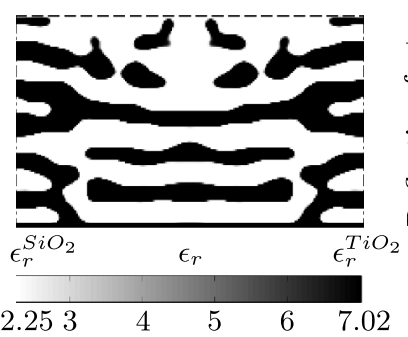

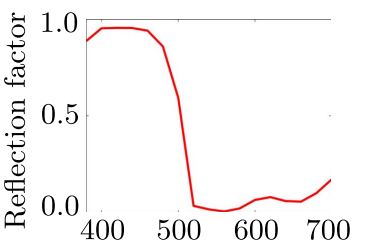

$\Phi=0.9944$
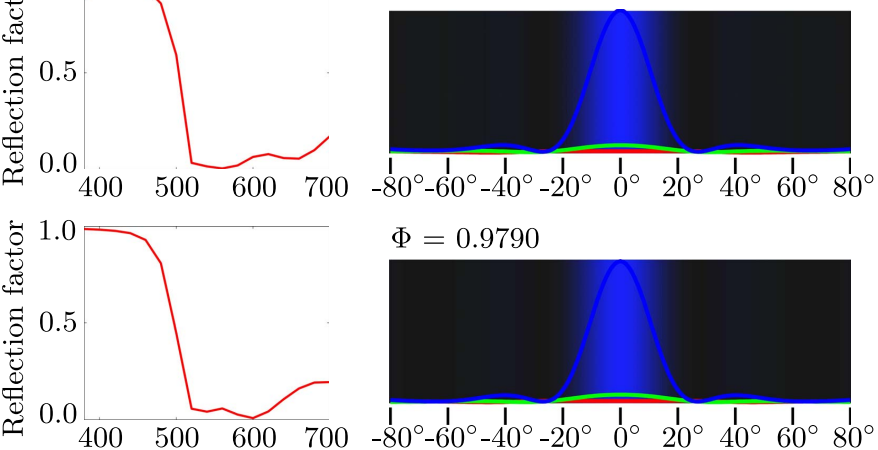

$\Phi=0.9790$
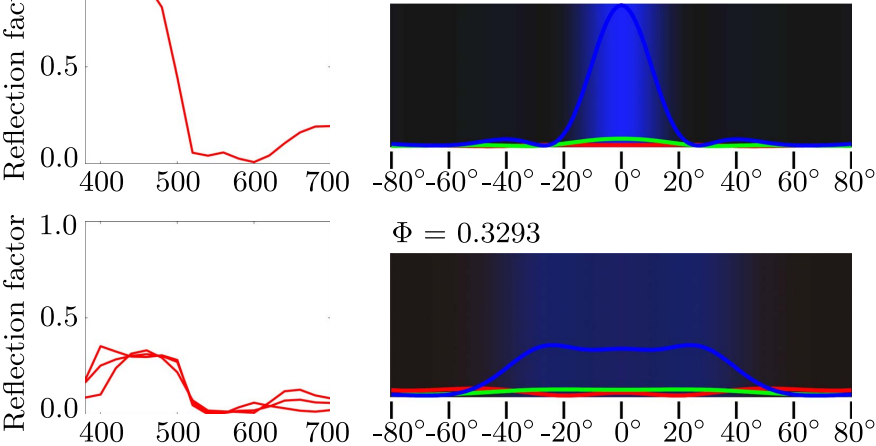

$\Phi=0.3293$
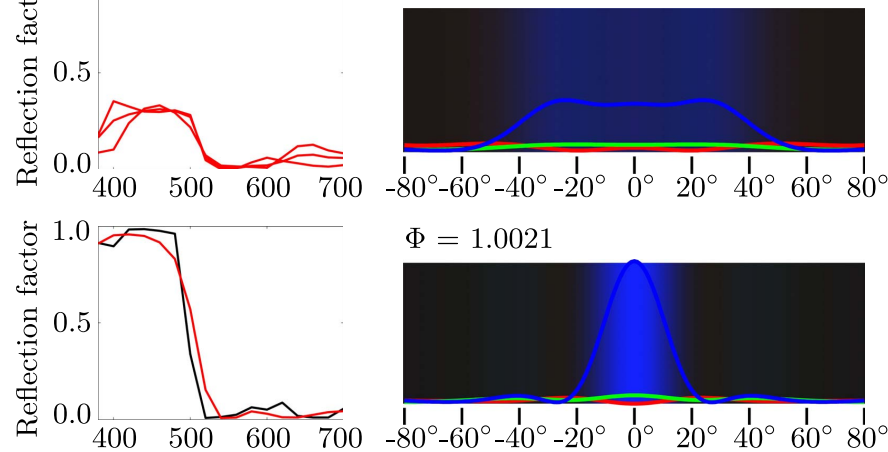

$\Phi=1.0021$
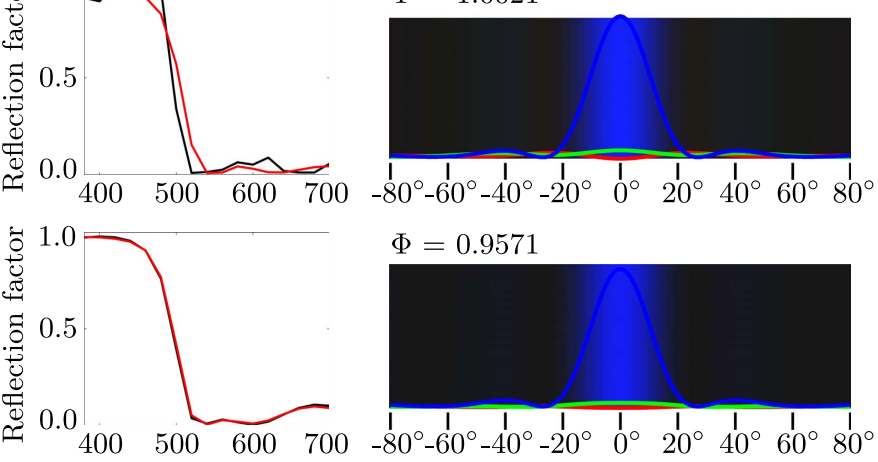

$\Phi=0.9571$
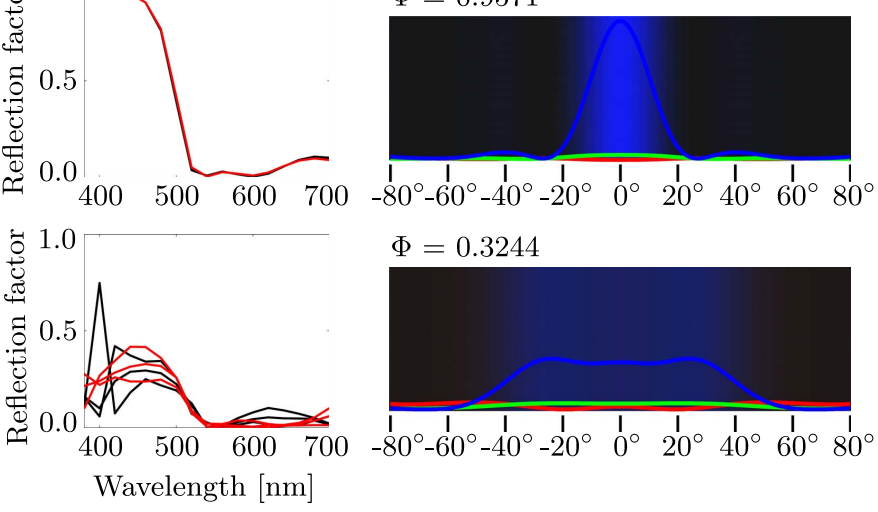

$\Phi=0.3244$

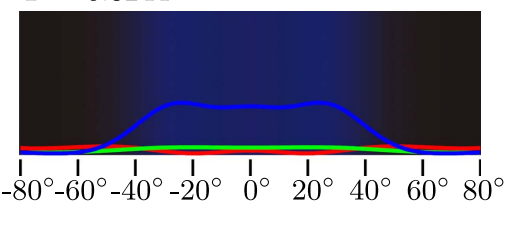

Fig. 7. Color optimization for combined $E_{z}$ and $H_{z}$ polarization. (a)-(c) Structures optimized for $H_{z}$ polarization corresponding to those optimized for $E_{z}$ in Figs. 4(c), 5(c), and 6(c), respectively. (d)-(f) Similar structures optimized for combined $E_{z}$ and $H_{z}$ polarization. Red curves in the reflection plots $\overline{c o r r}$ spond to $\overline{H_{z}}$ polarization and black to $E_{z}$. 
specular direction $\left(0^{\circ}\right)$ with free topology; (2) optimizing the color in the specular direction $\left(0^{\circ}\right)$ with the geometry restricted to simple (Bragg grating-like) layerings; and (3) optimizing the color in the angular range $\left(-30^{\circ}\right.$ to $\left.+30^{\circ}\right)$ with free topology. In practise, the optimizations for angular intervals are only optimized for $N$ discrete angles (here $N=5$, which is reduced to $N=3$ for symmetry reasons). For the three formulations, we optimize structured surfaces to produce the six main colors: red, green, blue, magenta, yellow, and cyan as well as black and white. The results are shown in Figs. 4, 5, and 6 , respectively. The result of each individual optimization is presented as a bitmap picture of the optimized topology (left panel), reflection spectra for each output angle and polarization (middle panel), and an angular color spectrum with background color corresponding to the perceived color and individual curves for the three basis colors: red, green, and blue (right panel). The $\Phi$ values given for each example denotes the color intensity as defined in Eq. (12).

The optimization problem is highly nonconvex, and convergence to a global optimum cannot be guarantied. However, a continuation strategy runs with different starting guesses, and previous experience make us confident that very good solutions are found. In order to perform a sanity check of the obtained results, we compare objective functions for the three cases in Table 1 . As expected, the reduced design freedom, as represented by the layered structures in Fig. 5, cannot in all cases produce the same intensity as the freely optimized structures from Fig. 4. Likewise, the structures optimized for a wider angular interval (Fig. 6) show much lower angular color intensity. It also can be seen that the color intensities for green and magenta are lower than for the others colors, especially for the simple layered structures. This also is to be expected since the forming of green and magenta requires a stop band and a pass band, respectively, whereas all other colors can be obtained by simpler low or high-pass filters.
From the comparisons above, it is clear that freely optimized structures in Fig. $\underline{4}$ are indeed better than simple layered ones in Fig. 5. With the increased design freedom, it is possible not only to create simple $1 \mathrm{D}$ gratings with specific pass and stop behavior but also to scatter light away from the observation point or alternatively to create more complex structures that may improve transmission of frequencies unwanted in the reflection spectrum. Both possibilities may lead to improved color response at the observation point.

The red, green, and magenta designs perform surprisingly bad for the simple layered designs in Fig. 5. However, these are also the designs that resemble layered structures the least for the full design freedom in Fig. 4 . Hence, it is concluded that complex structures seen in Figs. 4(a), 4(b), and 4(e) are indeed needed to ensure intense and pure colors.

For the structures optimized for broad angular response in Fig. $\underline{6}$, the simple layered structures become highly suboptimal since they only reflect light back in a limited angular range. Instead all the optimized designs exhibit rather complex structures that are difficult to interpret. They all exhibit a kind of directional photonic crystal structure, and it is observed that the periodicity varies with wavelength. For example, the structure optimized for red color generation (long wavelength) has larger and more distant inclusions than the structure optimized for blue color generation (short wavelength). For the chosen cell size, the angle for first-order diffraction for red light is $43^{\circ}$, and for blue light it is $27^{\circ}$. Hence, when optimizing color for the angular interval $\left[-30^{\circ}, 30^{\circ}\right]$, red will have an advantage since it does not need to include second-order diffraction effects in order to reflect evenly in a broad angular interval. This observation may explain the superiority of the red design in Fig. $\underline{6}$ compared to the other colors.

The previous designs were all obtained for a single polarization. In Fig. 7 we demonstrate the effect of polarization (a)

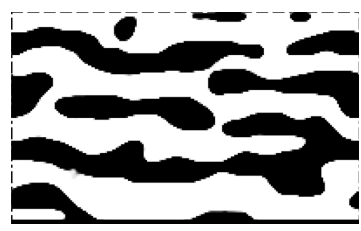

(b)

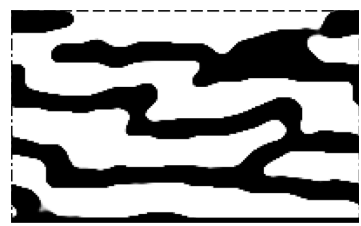

(c)

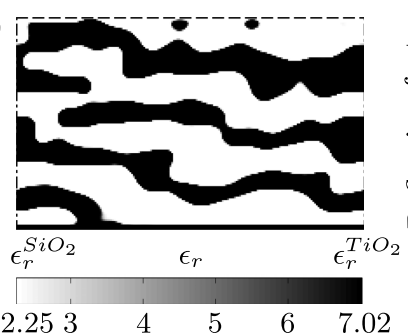

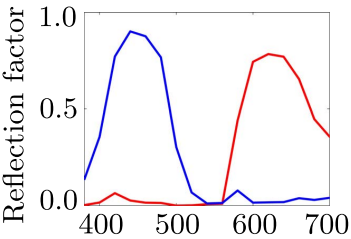

$\Phi=0.8916$
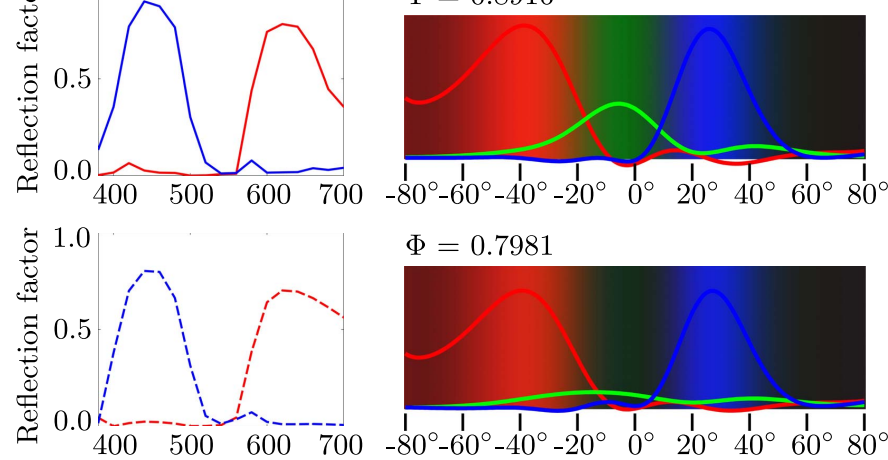

$\Phi=0.7981$
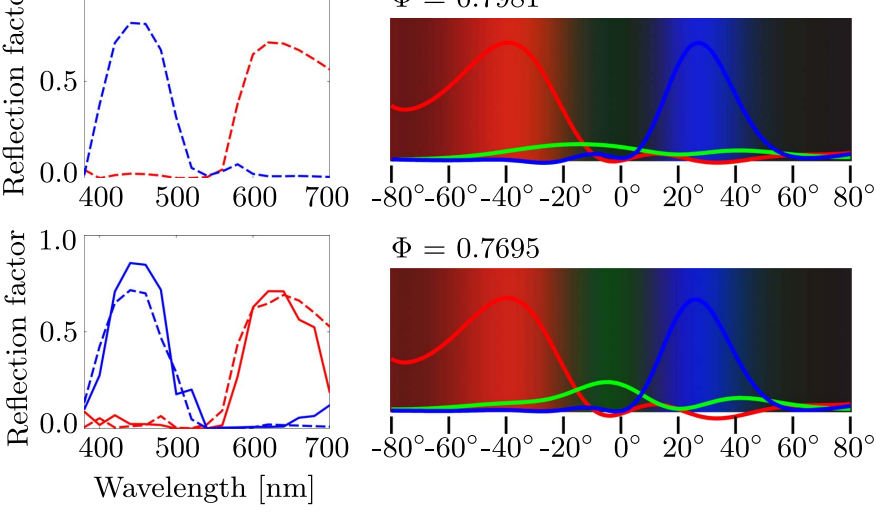

$\Phi=0.7695$

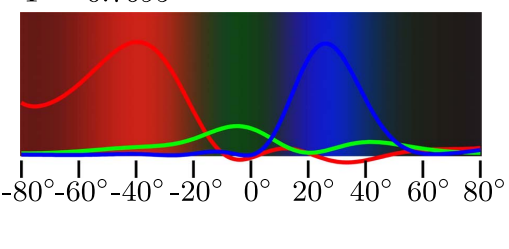

Fig. 8. Optimization for prescribed iridescent colors. Red color $[1,0,0]$ intensity is optimized for $-43^{\circ}$ observer direction, and blue color intensity $[0,0,1]$ is optimized for $27^{\circ}$ observer direction. Designs (a)-(c) correspond to optimized structures for $E_{z}, H_{z}$, and combined $E_{z}$ to $H_{z}$ polarization, respectively. Red curves correspond to the reflection in the $-43^{\circ}$ direction and blue to the reflection in the $27^{\circ}$ direction. 
on structures optimized for blue color generation. Figures $7(\mathrm{a})-7(\mathrm{c})$ show the designs optimized for $H_{z}$ polarization, which can be compared to the similar ones optimized for $E_{z}$ polarization in Figs. $\underline{4(\mathrm{c})}, \underline{5(\mathrm{c})}$, and $\underline{6(\mathrm{c})}$, respectively. These designs appear not to be qualitatively much different from each other, indicating that polarization is not a big issue from a design view point. Figures $7(\mathrm{~d})-7(\mathrm{f})$ show the corresponding designs optimized for both polarizations simultaneously. Color intensities only drop insignificantly, indicating that it is quite easy to design polarization-independent structural color surfaces using the developed methodology.

The final example considers the design of a surface with angular-dependent (iridescent) color properties. Again, the incoming light is perpendicular to the surface, but we require the reflected colors to be red in the $-43^{\circ}$ direction and blue in the $27^{\circ}$ direction. The optimized structures for $E_{z}$ and $H_{z}$ polarization are shown in Figs. $\underline{8(\mathrm{a})}$ and $8(\mathrm{~b})$, respectively. It is observed that large red and blue color intensities can be obtained in the prescribed directions, and that the color is vague green in between. If the green color is unwanted, one may prescribe black for the zero-degree reflection angle, making the optimization generate a structure that transmits more of the green color instead of reflecting it. Figure 8(c) shows the same optimization performed for simultaneous $\overline{E_{z}}$ and $H_{z}$ polarization. Again, requirements for nonpolarized response do not alter the obtainable color intensities significantly. The optimized structures are difficult to interpret; however, slanted layered structures can be seen that help to redirect the waves in the desired directions.

\section{CONCLUSION}

We have proposed a systematic procedure for generating nanostructured surfaces with prescribed structural color response. The procedure is demonstrated for a number of cases, including generation of arbitrary colors in narrow- or wideangle intervals as well as for angular-dependent or iridescent color generation. The developed software is general and can be applied to any kind of material properties. For the examples, we considered a $\mathrm{TiO}_{2} / \mathrm{SiO}_{2}$ nanostructure on a $\mathrm{TiO}_{2}$ substrate, but the procedure also can handle single material structures in air. In this case, manufacturing constraint must be included to prevent nonconnected parts as, for example, seen in [12]. In the present paper, we allow full design freedom, which results in strong color effects even for thin design domains. If complex nanostructures are not allowed by the manufacturing process, additional constraints must be imposed, inevitably resulting in decreased color-generation performance. The proposed procedure can find many applications. It may be used for the optimal study of structural color surfaces found in nature, extended to three dimensions, allowing for more advanced color effects, and extended to plasmonic design problems for color generation (cf. [30]) or thin film solar cells [31, $\underline{32}]$.

\section{APPENDIX A: DERIVATION OF IMPRESSED SURFACE ELECTRIC CURRENT DENSITY}

On the incoming wave boundary $\Gamma_{i}$, we have the boundary condition [20, p. 10]

$$
\hat{\mathbf{n}} \times\left(\mathbf{H}_{2}-\mathbf{H}_{1}\right)=\mathbf{J}=\hat{\mathbf{z}} J_{s z},
$$

where $\hat{\mathbf{n}}=\hat{\mathbf{x}} \hat{n}_{x}+\hat{\mathbf{y}} \hat{n}_{y}$ is the downward pointing normal to $\Gamma_{i}$, and $\mathbf{H}_{2} / \mathbf{H}_{1}$ are the magnetic fields existing just above/below $\Gamma_{i}$, respectively, and $J_{s z}$ is the impressed surface electric current density, which only has a $z$ component since the $H$ fields and the normal are perpendicular to $\hat{\mathbf{z}}$. For simple media, it holds that $\mathbf{H}=1 /\left(j \omega \mu_{r} \mu_{0}\right) \nabla \times \mathbf{E}$, and since we have defined $\mathbf{E}=\hat{\mathbf{z}} E_{z}$, this in turn leads to

$$
\nabla \times \mathbf{E}=\hat{\mathbf{x}} \frac{\partial E_{z}}{\partial y}-\hat{\mathbf{y}} \frac{\partial E_{z}}{\partial x}
$$

The cross-product $\hat{\mathbf{n}} \times \mathbf{H}$ from this can be found to be

$$
\hat{\mathbf{n}} \times \mathbf{H}=\frac{1}{j \omega \mu_{r} \mu_{0}}\left(\hat{n}_{y} \frac{\partial E_{z}}{\partial y}+\hat{n}_{x} \frac{\partial E_{z}}{\partial y}\right)=\frac{1}{j \omega \mu_{r} \mu_{0}} \hat{\mathbf{n}} \cdot\left(\nabla E_{z}\right),
$$

where $\mu_{r}$ is assumed constant around $\Gamma_{i}$. Combining Eqs. (A1) and (A3), the expression

$$
\hat{\mathbf{n}} \cdot\left(\nabla E_{z 2}-\nabla E_{z 1}\right)=\hat{\mathbf{z}} j \omega \mu_{r} \mu_{0} J_{s z}
$$

is obtained, where $E_{z 1}, E_{z 2}$ are the $z$ components of $\mathbf{E}_{1}, \mathbf{E}_{2}$ related to $\mathbf{H}_{1}, \mathbf{H}_{2}$, respectively. Since we cannot expect to excite a wave in one direction without exciting one in the opposite direction as well, we define the fields that should be excited by $J_{s z}$ to be

$$
\begin{aligned}
& \mathbf{E}_{1}=\hat{\mathbf{z}} E_{z 0} \exp \left(-j k_{0} \sqrt{\mu_{r} \epsilon_{r}} \hat{\mathbf{k}}_{1} \cdot \mathbf{r}\right), \\
& \mathbf{E}_{2}=\hat{\mathbf{z}} E_{z 0} \exp \left(-j k_{0} \sqrt{\mu_{r} \epsilon_{r}} \hat{\mathbf{k}}_{2} \cdot \mathbf{r}+j \phi\right),
\end{aligned}
$$

where $\phi$ is a constant chosen such that $\mathbf{E}_{1}, \mathbf{E}_{2}$ match on $\Gamma_{i}$. Assuming that $\Gamma_{i}$ is parallel to the $y$ direction and defining $\hat{\mathbf{k}}_{1} \equiv \hat{\mathbf{k}}=\left(\hat{k}_{x}, \hat{k}_{y}\right)^{T}$ such that the incoming wave is equivalent to Eq. (3), then $\hat{\mathbf{k}}_{2}=\left(-\hat{k}_{x}, \hat{k}_{y}\right)^{T}$. This in turn means that $\nabla E_{z 1}=-j k_{0} \sqrt{\mu_{r} \epsilon_{r}} \hat{\mathbf{k}} E_{z 1}$ and $\nabla E_{z 2}=-j k_{0} \sqrt{\mu_{r} \epsilon_{r}} \hat{\mathbf{k}}_{2} E_{z 2}$, which, combined with Eq. (스), leads to

$$
J_{s z}=\frac{j k_{0} \sqrt{\mu_{r} \epsilon_{r}}}{j \omega \mu_{0} \mu_{r}} \hat{\mathbf{n}} \cdot\left(\hat{\mathbf{k}}_{1} E_{z 1}-\hat{\mathbf{k}}_{2} E_{z 2}\right)
$$

Since the two electrical fields are the same on $\Gamma_{i}$ and $\hat{\mathbf{n}}=$ $(1,0)$ for $\Gamma_{i}$ along the $y$ axis, the surface electric current density exciting the wave in Eq. (3) (and also a wave in the other direction) can then be found as

$$
J_{s z}=2 \hat{k}_{x} \sqrt{\frac{\epsilon_{0} \epsilon_{r} \mu_{r}}{\mu_{0}}} E_{z, 1}
$$

\section{ACKNOWLEDGMENTS}

We are grateful for the support from the Danish National Technology Foundation through the projects NANOPlast and ODAAS and the Villum Foundation through the NextTop project.

\section{REFERENCES}

1. M. Kolle, Photonic Structures Inspired by Nature (Springer, 2011). 
2. S. Kinoshita and S. Yoshioka, "Structural colors in nature: the role of regularity and irregularity in the structure," Chem. Phys. Chem. 6, 1442-1459 (2005).

3. M. Crne, V. Sharma, J. Blair, J. O. Park, C. J. Summers, and M. Srinivasarao, "Biomimicry of optical microstructures of Papilio palinurus," Europhys. Lett. 93, 14001 (2011).

4. S. Kinoshita, S. Yoshioka, and J. Miyazaki, "Physics of structural colors," Rep. Prog. Phys. 71, 076401 (2008).

5. R. T. Lee and G. S. Smith, "Detailed electromagnetic simulation for the structural color of butterfly wings," Appl. Opt. 48, 4177-4190 (2009).

6. M. A. Steindorfer, V. Schmidt, M. Belegratis, B. Stadlober, and J. R. Krenn, "Detailed simulation of structural color generation inspired by the Morpho butterfly," Opt. Express 20, 2148521494 (2012).

7. N. Okada, D. Zhu, D. Cai, J. B. Cole, M. Kambe, and S. Kinoshita, "Rendering Morpho butterflies based on high accuracy nanooptical simulation,” J. Opt. 42, 25-36 (2013).

8. A. Saito, "Material design and structural color inspired by biomimetic approach," Sci. Technol. Adv. Mat. 12, 064709 (2011).

9. K. Kumar, H. Duan, R. S. Hegde, S. C. W. Koh, J. N. Wei, and J. K. W. Yang, "Printing colour at the optical diffraction limit," Nat. Nanotechnol. 7, 557-561 (2012).

10. D. C. Dobson, "Optimal design of periodic antireflective structures for the Helmholtz equation," Euro. J. Appl. Math. 4, 321-339 (1993).

11. K. Fuchi, A. R. Diaz, E. Rothwell, R. Ouedraogo, and A. Temme, "Topology optimization of periodic layouts of dielectric materials," Struct. Multidiscip. Optim. 42, 483-493 (2010).

12. K. S. Friis and O. Sigmund, "Robust topology design of periodic grating surfaces," J. Opt. Soc. Am. B 29, 2935-2943 (2012).

13. M. P. Bendsøe and O. Sigmund, Topology OptimizationTheory, Methods and Applications (Springer-Verlag, 2004).

14. M. P. Bendsøe and N. Kikuchi, "Generating optimal topologies in structural design using a homogenization method," Comput. Methods Appl. Mech. Eng. 71, 197-224 (1988).

15. J. S. Jensen and O. Sigmund, "Topology optimization of photonic crystal structures: a high-bandwidth low-loss T-junction waveguide," J. Opt. Soc. Am. B 22, 1191-1198 (2005).

16. J. S. Jensen and O. Sigmund, "Topology optimization for nanophotonics," Laser Photon. Rev. 5, 308-321 (2011).

17. Y. Elesin, B. S. Lazarov, J. S. Jensen, and O. Sigmund, "Time domain topology optimization of 3D nanophotonic devices," Photon. Nanostruct. Fundam. Appl., doi: 10.1016/j.photonics.2013.07.008 (2013).
18. A. Erentok and O. Sigmund, "Topology optimization of subwavelength antennas," IEEE Trans. Anten. Propag. 59, 58-69 (2011).

19. A. R. Diaz and O. Sigmund, "A topology optimization method for design of negative permeability metamaterials," Struct. Multidiscip. Optim. 41, 163-177 (2010).

20. J. Jin, The Finite Element Method in Electromagnetics 2nd ed. (Wiley, 2002).

21. CIE, "Selected colorimetric tables," http://www.cie.co.at.

22. R. S. Berns, F. W. Billmeyer, and M. Saltzman, Billmeyer and Saltzman's Principles of Color Technology (Wiley-Interscience, 2000).

23. A. Saito, Y. Miyamura, Y. Ishikawa, J. Murase, M. Akai-Kasaya, and Y. Kuwahara, "Reproduction, mass production, and control of the Morpho butterfly's blue," in Advanced Fabrication Technologies for Micro/Nano Optics and Photonics II, T. J. Suleski, W. V. Schoenfeld, and J. J. Wang, eds. (SPIE, 2009), Vol. 7205, p. 720506.

24. A. Saito, M. Yonezawa, J. Murase, S. Juodkazis, V. Mizeikis, M. Akai-Kasaya, and Y. Kuwahara, "Numerical analysis on the optical role of nano-randomness on the Morpho butterfly's scale," J. Nanosci. Nanotechnol. 11, 2785-2792 (2011).

25. J. A. Andkjær, S. Nishiwaki, T. Nomura, and O. Sigmund, "Topology optimization of grating couplers for the efficient excitation of surface plasmons," J. Opt. Soc. Am. B 27, 1828-1832 (2010).

26. O. Sigmund, "On the usefulness of non-gradient approaches in topology optimization," Struct. Multidisc. Optim. 43, 589-596 (2011).

27. O. Sigmund, "Manufacturing tolerant topology optimization," Acta Mech. Sinica 25, 227-239 (2009).

28. F. Wang, B. S. Lazarov, and O. Sigmund, "On projection methods, convergence and robust formulations in topology optimization," Struct. Multidisc. Optim. 43, 767-784 (2011).

29. K. Svanberg, "The method of moving asymptotes-a new method for structural optimization," Intern. J. Numer. Meth. Eng. 24, 359-373 (1987).

30. K. Kumar, H. Duan, R. S. Hegde, S. C. W. Koh, J. N. Wei, and J. K. W. Yang, "Printing colour at the optical diffraction limit," Nat. Nanotechnol. 7, 557-561 (2012).

31. M. B. Dühring and O. Sigmund, "Optimization of extraordinary optical absorption in plasmonic and dielectric structures," J. Opt. Soc. Am. B 30, 1154-1160 (2013).

32. X. Sheng, S. G. Johnson, J. Michel, and L. C. Kimerling, "Optimization-based design of surface textures for thin-film Si solar cells," Opt. Express 19, A841-A850 (2011). 